\title{
Risk factors for suicide among patients with schizophrenia: a cohort study focused on cerebrospinal fluid levels of homovanillic acid and 5-hydroxyindoleacetic acid
}

\author{
This article was published in the following Dove Press journal: \\ Neuropsychiatric Disease and Treatment \\ II July 2016 \\ Number of times this article has been viewed
}

\section{Daniel Neider \\ Leif $\mathrm{H}$ Lindström \\ Robert Bodén}

Department of Neuroscience, Psychiatry, Uppsala University, Uppsala University Hospital, Uppsala, Sweden
Correspondence: Daniel Neider Department of Neuroscience, Psychiatry, Uppsala University, Uppsala University Hospital, Entrance 10, 75I85 Uppsala, Sweden

Fax +46 I8 260065

Email daniel.neider@akademiska.se
Background: The objective of this study was to investigate the association between 5-hydroxyindoleacetic acid (5-HIAA) and homovanillic acid (HVA) in cerebrospinal fluid (CSF), bullying, and later suicide among patients with schizophrenia.

Methods: Ninety-nine patients with schizophrenia were included. Correlations of clinical factors, 5-HIAA and HVA, and later suicide were investigated.

Results: Twelve patients committed suicide (12\%) during a 28-year follow-up period. Later suicide was correlated to bullying in childhood $(P=0.02)$ and a lower quotient of HVA/5-HIAA in CSF $(P<0.05)$.

Conclusion: Suicide in schizophrenia is related to childhood exposedness and CSF neurotransmitter levels.

Keywords: psychosis, risk factors, bullying, HVA, 5-HIAA, CSF

\section{Introduction}

Suicide is a common and tragic outcome among patients with schizophrenia, with a lifetime risk being $\sim 5 \%$. ${ }^{1}$ Some risk factors for suicide in individuals with schizophrenia are similar to those in the general population. Higher education, older age at onset of symptoms,${ }^{2}$ male sex, substance abuse, and number of admissions have been associated with increased suicide risk in patients with schizophrenia. ${ }^{3}$ Bullying during childhood and adolescence has been related to increased risk of suicidal behavior in young life. ${ }^{4}$ A meta-analysis indicates that among patients with schizophrenia, affective symptoms such as agitation (or motor restlessness) and sense of worthlessness and hopelessness increase the risk of suicide, while hallucinations are associated with a reduced risk. ${ }^{5}$

Much interest has been focused on the possible impact of the neurotransmitters dopamine and serotonin in suicidal behavior, ${ }^{6-8}$ mostly by measurement of neurotransmitters 5-hydroxyindoleacetic acid (5-HIAA) and homovanillic acid (HVA) in cerebrospinal fluid (CSF), but mainly in mixed samples or mainly patients with affective disorders.

Results from studies on HVA and 5-HIAA in patients with schizophrenia spectrum disorders are contradictory and many are hampered by short follow-up periods. ${ }^{9,10}$ Patients with a diagnosis of schizophrenia have been shown to have low HVA and normal 5-HIAA levels compared to healthy volunteers, ${ }^{11}$ and patients with a poor function (frequency of social contacts, employment duration, symptomatology and duration of rehospitalization) on a 5-year-up follow-up were found to have a low quotient of HVA/5-HIAA. ${ }^{12}$ 
The aim of the present study was to explore the relationship between various clinical, sociodemographic, and biological markers and later suicide.

\section{Methods}

\section{Setting and participants}

This is a cohort study and has been previously described in detail. ${ }^{12}$ Patients were consecutively admitted to a ward for psychotic patients at the University Hospital in Uppsala, Sweden, between 1979 and 1986. Exclusion criteria included alcohol or drug abuse, present neuroleptic medication, and severe somatic disease. A total of 99 patients ( schizophrenia, $\mathrm{n}=91$; schizophreniform disorder, $\mathrm{n}=1$; schizoaffective disorder, $n=7$ ) were thoroughly examined including psychiatric history of relatives, rating with the Comprehensive Psychopathological Rating Scale (CPRS), ${ }^{13}$ and CSF HVA, 5-HIAA at admission. Fifty-eight patients (59\%) were admitted for the first time and had never been treated with neuroleptics. The previously known 41 patients (41\%) were readmitted but without current antipsychotic medication. The study was approved by the Ethical Review Board of Uppsala University, and oral consent was given by all patients.

\section{Lumbar puncture procedure}

CSF was sampled by lumbar puncture before antipsychotic treatment was initiated (L4-L5); $18 \mathrm{~mL}$ of CSF was collected with a $0.9 \mathrm{~mm}$ needle and the sample was immediately centrifuged at $1,000 \times g$ for 10 minutes at ambient temperature, decanted, mixed well to avoid fractionating, and then divided into aliquots of $1 \mathrm{~mL}$. Within 20 minutes of the sampling procedure, $\mathrm{CSF}$ was stored at $-80^{\circ} \mathrm{C}$ until assayed. Subjects were not allowed to smoke before the procedure.

\section{Assay for HVA and 5-HIAA}

The assays were performed according to the procedure described by Swahn et $\mathrm{al}^{14}$ using an LKB 2091 combined gas chromatograph-mass spectrometer equipped with a multiple ion detector. The results were adjusted for sex and height. ${ }^{11}$

\section{Clinical and sociodemographic factors}

The following CPRS items where literature indicates association with suicide were evaluated: hallucinations (including commenting voices, other auditory hallucinations, visual hallucinations, other hallucinations including taste, smell, and tactile sensations), sadness, labile emotions, hostility, overactivity, and agitation. The evaluated symptoms were dichotomized into low/high values indicating pathological symptoms or not ( 0 vs $1-3$ points on the CPRS rating scale). The various modalities of hallucinations (commenting voices, other voices, visual, tactile, smell, and taste) as well as increased motoric activity and agitation were gathered as one item. Patients and close relatives were interviewed regarding sociodemographic data including exposure to bullying during childhood.

\section{Outcome}

Suicide was the primary outcome. To identify the patients who died during the follow-up period, information was obtained from the Cause of Death Register, which is a nationwide register and covers more than $99 \%$ of all deaths occurring in Sweden. We identified those patients who died by committing suicide or due to undetermined cause according to the International Classification of Diseases 8, 9, and 10 (E950-E959, E950-959, and X60-X64, respectively). When the cause of death was ambiguous in the registers, the psychiatric records were reviewed for additional information. Sudden undetermined cause of death at young age or an accident where suicide could be a possible explanation was also defined as suicide $(n=4)$. Index admission was defined at the time of lumbar puncture, and patients were followed up until March 2012 or until the time of death.

\section{Statistics}

As the sample size was small, continuous data were analyzed with Mann-Whitney $U$-test, and correlations were calculated with Pearson's coefficient. For dichotomized variables, chisquare test was used.

\section{Results}

The total follow-up time was 2,461 person years. The median follow-up time from index admission was 28 years, with a mean of 25 years. The mean age at admission was 27 years (range 18-48 years).

Of the 99 patients in the cohort, 26 died during the follow-up period. Of the 26 patients, eight committed suicide according to the Cause of Death Register. Four undetermined deaths were defined as probable suicides and included in the suicide group (two patients [both aged 31 years] with sudden death of unknown cause, one patient [aged 46 years] found dead at home due to pulmonary edema, and one patient [aged 45 years] died in a fire). In total, deaths in 12 (12\%) patients were defined as definite or probable suicides. Mean and median age at suicide was 34 and 31 years, respectively. Mean time from index admission to suicide was 9 years and median time was 5 years. Of the patients committing suicide, eight were drug-naïve and four were without 
Table I Sociodemographics, symptoms, and 5-HIAA and HVA in relation to suicide in a cohort of patients with schizophrenia

\begin{tabular}{lll}
\hline & $\begin{array}{l}\text { Suicide } \\
\mathbf{n}=\mathbf{1 2}\end{array}$ & $\begin{array}{l}\text { Non-suicide } \\
\mathbf{n}=\mathbf{8 7}\end{array}$ \\
\hline $\begin{array}{l}\text { Sociodemographic } \\
\text { Bullying }\end{array}$ & $6 / 1 \mathrm{I}$ & $17 / 77^{*}$ \\
Sex male vs female & 9 vs 3 & 56 vs 3I \\
Age at inclusion, years (SD) & $22.0(4.1)$ & $24.3(4.7)$ \\
DUP $<$ I year & $8 / 12$ & $46 / 85$ \\
Education (graduated high school) & $6 / 12$ & $44 / 87$ \\
CPRS parameters & & \\
Hallucinations & $9 / 12$ & $43 / 83$ \\
Agitation/overactivity & $5 / 12$ & $33 / 87$ \\
Apparent sadness & $5 / 12$ & $33 / 87$ \\
Labile emotions & $2 / 12$ & $7 / 87$ \\
Hostility & $1 / 12$ & $5 / 87$ \\
Biological markers & & \\
HVA, nmol/mL (SD) & $140(72)$ & $192(108)$ \\
5-HIAA, nmol/mL (SD) & $1 / 12(48)$ & $1 / 5(4 I)$ \\
HVA/5-HIAA quotient (SD) & $1.2(0.9)$ & $1.8(0.8)^{*}$ \\
\hline
\end{tabular}

Notes: $* P<0.05$; CPRS items are tested with chi-square test, HVA and 5-HIAA with Mann-Whitney $U$-test. CPRS items dichotomized as present or not.

Abbreviations: 5-HIAA, 5-hydroxyindoleacetic acid; HVA, homovanillic acid; SD, standard deviation; CPRS, Comprehensive Psychopathological Rating Scale; DUP, duration of untreated psychosis.

antipsychotic treatment for $2-7$ weeks at the time of index admission. In the reference group of 87 patients, 49 were drug-naïve (56\%) and the remaining patients were drug-free for a mean of 7.5 weeks (range 2-26 weeks) at the time of index admission.

The results are summarized in Table 1. There was no difference in HVA or 5-HIAA levels, but the HVA/5-HIAA quotient was significantly lower in the suicide group. The number of patients who reported bullying victimization was significantly higher in the suicide group. There was no correlation between the sociodemographic variables sex, duration of untreated psychosis, education, and suicide. None of the clinical symptoms (hallucinations, sadness, labile emotions, hostility, overactivity, and agitation) differed between the two groups. There was no correlation between bullying and HVA or 5-HIAA levels.

\section{Discussion}

The key result from this study of a nonmedicated cohort of patients with schizophrenia at index admission is that bullying during childhood and a low quotient of HVA/5-HIAA in CSF are associated with later suicide.

A strength of the present study is the homogenous cohort of patients with schizophrenia with a long follow-up period and no attrition. The majority of the patients were drug-naïve and the rest were unexposed to antipsychotics for 2-26 weeks prior to admission. There is a theoretical possibility that previous antipsychotic treatment may have influenced the
CSF levels of HVA and 5-HIAA. However, there were no obvious differences in previous antipsychotics exposure between the cases and controls. Limitations of this study are the small number of suicides, which hampers the analytical possibilities, and the lack of data on treatments and habits such as smoking and food habits during the years before index admission that may affect neurotransmitter levels. We have, however, no reason to believe that food and smoking habits differed between the two groups.

Lifetime risk of suicide among patients with schizophrenia has been estimated around $10 \%$, but newer data estimate the risk to be $\sim 5 \% .^{1}$ In our study, there was a higher incidence (12\%). This could be coincidental due to a relatively small sample size.

A review of suicidal ideation and attempts following exposure to bullying in the general population indicates increased risk. ${ }^{15}$ Increased risk of suicidal behavior and suicide was seen among both girls and boys after being exposed to bullying, but after controlling for conduct and depression symptoms this was only significant for girls. ${ }^{16} \mathrm{~A}$ bullying victim experiences the suffering of defeat and humiliation, which could lead to entrapment, hopelessness, depression, and suicidal behavior. ${ }^{4}$ To the best of the authors' knowledge, this has not been found in the case of schizophrenia before; hence, further studies are needed to see if this is a replicable observation.

An analysis of a large cohort of 385 schizophrenia spectrum psychosis patients did not show a correlation between the levels of CSF HVA, 5-HIAA, or HVA/5-HIAA quotient and suicide. ${ }^{9}$ However, this was a heterogeneous cohort of patients with psychosis.

Notably, a previous finding from the same cohort as the present study was that low function is also associated with a low HVA/5-HIAA quotient. ${ }^{12}$ The mechanism behind these observations remains to be elucidated. The correlation of low HVA/5-HIAA quotient to later suicide could be a chance finding. However, it may represent an imbalance between the dopaminergic and serotonergic systems associated with factors important for later suicidal behavior such as impulse control or mood regulation.

\section{Acknowledgment}

The authors wish to thank Hans Arinell for statistical support.

\section{Disclosure}

The authors report no conflicts of interest in this work.

\section{References}

1. Palmer BA, Pankratz VS, Bostwick JM. The lifetime risk of suicide in schizophrenia: a re-examination. Arch Gen Psychiatry. 2005;62(3): 247-253. 
2. Reutfors J, Brandt L, Jönsson EG, Ekbom A, Sparén P, Ösby U. Risk factors for suicide in schizophrenia: findings from a Swedish populationbased case-control study. Schizophr Res. 2009;108:231-237.

3. Popovic D, Benabarre A, Crespo JM, et al. Risk factors for suicide in schizophrenia: systematic review and clinical recommendations. Acta Psychiatr Scand. 2014;130(6):418-426.

4. Meltzer H, Vostanis P, Ford T, Bebbington P, Dennis MS. Victims of bullying in childhood and suicide attempts in adulthood. Eur Psychiatry. 2011;26:498-503.

5. Hawton K, Sutton L, Haw C, Sinclair J, Deeks JJ. Schizophrenia and suicide: systematic review of risk factors. Br J Psychiatry. 2005;187: 9-20.

6. Sher L, Mann JJ, Traskman-Bendz L, et al. Lower cerebrospinal fluid homovanillic acid levels in depressed suicide attempters. $J$ Affect Disord. 2006;90(1):83-89.

7. Asberg M, Träskman L, Thoren P. 5-HIAA in cerebrospinal fluid. A biochemical suicide predictor? Arch Gen Psychiatry. 1976;33(10): 1193-1197.

8. Engström G, Alling C, Blennow K, Regnéll G, Träskman-Bendz L. Reduced cerebrospinal HVA concentrations and HVA/5-HIAA ratios in suicide attempters. Monoamine metabolites in 120 suicide attempters and 47 controls. Eur Neuropsychopharmacol. 1999;9(5):399-405.

9. Carlborg C, Jokinen J, Nordström AL, Jönsson EG, Nordström P. CSF 5-HIAA, attempted suicide and suicide risk in schizophrenia spectrum psychosis. Schizophr Res. 2009;112(1-3):80-85.
10. Banki CM, Arató M, Papp Z, Kurcz M. Biochemical markers in suicidal patients. Investigations with cerebrospinal fluid amine metabolites and neuroendocrine tests. J Affect Disord. 1984;6(3-4):341-350.

11. Lindström LH. Low HVA and normal 5HIAA CSF levels in drug-free schizophrenic patients compared to healthy volunteers: correlations to symptomatology and family history. Psychiatry Res. 1985;14(4): 265-273.

12. Wieselgren I, Lindström LH. CSF-levels of HVA and 5-HIAA in drug-free schizophrenic patients and healthy controls; a prospective study focused on their predictive value for outcome in schizophrenia. Psychiatry Res. 1998;81(2):101-110.

13. Asberg M, Montgomery SA, Perris C, Schalling D, Sedvall G. A comprehensive psychopathological rating scale. Acta Psychiatr Scand Suppl. 1978;(271):5-27.

14. Swahn C-G, Sandgärde B, Wiesel F-A, Sedvall G. Simultaneous determination of the three major monoamine metabolites in brain tissue and body fluids by a mass fragmentographic method. Psychopharmacology. 1976;48(2):147-153.

15. Kim YS, Leventhal B. Bullying and suicide. A review. Int J Adolesc Med Health. 2008;20(2):133-154.

16. Klomek AB, Sourander A, Niemelä S, et al. Childhood bullying behaviors as a risk for suicide attempts and completed suicides: a population-based birth cohort study. J Am Acad Child Adolesc Psychiatry. 2009;48(3): 254-261.
Neuropsychiatric Disease and Treatment

\section{Publish your work in this journal}

Neuropsychiatric Disease and Treatment is an international, peerreviewed journal of clinical therapeutics and pharmacology focusing on concise rapid reporting of clinical or pre-clinical studies on a range of neuropsychiatric and neurological disorders. This journal is indexed on PubMed Central, the 'PsycINFO' database and CAS,

\section{Dovepress}

and is the official journal of The International Neuropsychiatric Association (INA). The manuscript management system is completely online and includes a very quick and fair peer-review system, which is all easy to use. Visit http://www.dovepress.com/testimonials.php to read real quotes from published authors. 